\title{
Heart Coherence Training Combined with Back School in Patients with Chronic Non-specific Low Back Pain: First Pragmatic Clinical Results
}

\author{
Remko Soer • Dafne Vos $\cdot$ Bert Hofstra • \\ Michiel F. Reneman
}

(C) Springer Science+Business Media New York 2014

\begin{abstract}
The aim of this study was to explore on which variables a stress reduction program based on heart coherence can enhance the effects of a back school (BS) in patients with chronic non-specific low back pain and to explore possible moderators for treatment success. A retrospective explorative design was carried out with 170 patients with chronic non-specific low back pain. 89 Patients were admitted to BS and 81 patients were selected for BS and heart coherence training (BS-HCT). Six sessions of heart coherence were provided. At T0 (baseline) and T1 (discharge), the Numeric Rating Scale for pain (NRS pain), Roland Morris Disability Questionnaire (RMDQ), Pain Disability Index (PDI) and Rand-36 were administered in both groups. Both groups improved significantly on NRS pain, RMDQ, PDI and most of the Rand36 subscales. On physical functioning, the BS-HCT group improved significantly more than the BS group $(p=0.02)$ but not after Bonferroni correction. Significant moderate correlations $(r=0.39$ and $r=0.48)$ were found between the change of heart coherence and change of PDI and RMDQ respectively, but not with other variables. Baseline characteristics were not related to change on heart coherence. Providing HCT was more effective on physical
\end{abstract}

R. Soer · D. Vos - B. Hofstra - M. F. Reneman

Department of Rehabilitation Medicine, University Medical

Center Groningen, University of Groningen, Groningen,

The Netherlands

R. Soer $(\square)$

Groningen Spine Center, University Medical Center Groningen,

University of Groningen, Groningen, The Netherlands

e-mail: r.soer@umcg.nl

R. Soer

Expertise Center of Health, Social Care and Technology, Saxion

University of Applied Sciences, Enschede, The Netherlands functioning compared to a BS program. Change in heart coherence was related significantly to 2 out of 12 analyses. Placebo controlled and blinded studies are needed to confirm this. Characteristics of individuals who might benefit remain unknown. Evidence of this study is considered a level $\mathrm{C}$, because of its pragmatic clinical character.

Keywords Heart rate variability - Stress reduction . Low back pain · Back school $\cdot$ Emotion regulation

\section{Introduction}

Prevalence of non-specific chronic low back pain (CLBP) is estimated at approximately $20 \%$ in western European countries (Breivik et al. 2006). CLBP may affect both functioning and quality of life mediated by decrease in physical, emotional and social factors. Physiotherapists have accordingly shifted towards a biopsychosocial approach and there is evidence that a biomedical approach is insufficiently effective (Hay et al. 2005). There are multiple biopsychosocial treatment options which are currently evidence based including cognitive therapy combined with exercise therapy, operant approaches or contextual treatment such as mindfulness or acceptance and commitment therapy (ACT) (Keefe et al. 2004). The latter are relatively new therapies with proven effect and multiple guidelines advise to perform behavioral interventions (Bekkering et al. 2005; Tulder et al. 2010) for patients with CLBP.

Within the current western societal norms, factors such as high demanding work or financial crisis appear to considerably contribute to perceived stress. Studies show relations between those factors and the transition of acute towards chronic pain (Tunks et al. 2008; Turk and Okifuji 
2002). Central sensitization of pain modulation systems is one of the mediators in this transition (Van Oosterwijck et al. 2013). An increased activity of the limbic system was described in patients with chronic pain. The limbic system is involved in emotion and sympatic and parasympatic regulation (Heimer and Van Hoesen 2006). An increase of stress or pain experience was related to increased sympatic activity (Hallman and Lyskov 2012). An increase of sympatic activity, or decreased parasympatic activity affects heart rate variability (HRV) (Lane et al. 2009; Lehrer et al. 2003). HRV is defined as the variation in duration between successive heartbeats and is related to a range of emotional brain structures including prefrontal lobe and amygdale (Lane et al. 2009). A decreased HRV was related to decreased health on short and long term (Dekker et al. 2000; Stein and Kleiger 1999). Negative emotions, such as frustration, are known to lead to a decreased HRV (Agelink et al. 2002; Taelman et al. 2011). Positive emotions however, are hypothesized to lead to an increase of HRV, which in turn would lead to an increase in balance of the autonomic nerve system leading to better hormonal balance, immune system and more effective brain function (McCraty 2001). Additionally, sadness was related to an increase of high-frequency HRV but anger was not (van Middendorp et al. 2013).

A state in which there is balance between sympatic and parasympatic systems, has been described as coherency (McCraty et al. 2009). Heart coherence is defined as the state in which oscillation of HRV is symmetric and follows a sine curve (McCraty 2001). Factors including stress or insufficient coping are suggested to be associated with an incoherent pattern. Relaxation, appreciation, focussing on body signals such as breathing and heart rate is suggested to be associated with coherency (McCraty and Tomasino 2006).

While there is conflicting evidence on the exact relations and mediating mechanisms between sympatic, parasympatic activity and HRV (Goedhart et al. 2008; Houtveen et al. 2012), there appear some lower and higher quality studies that heart coherence training (HCT) appears effective for a decrease in stress, tension, fatigue, pain, hypertension and an increase in functioning and health in different patient populations in which stress is an important limiting factor (Hallman et al. 2011; Hassett et al. 2007; Lemaire et al. 2011). For physiotherapists working in primary or secondary care, there are only a limited number of stress reduction protocols available. While many physiotherapists have adopted the biopsychosocial model, clear translation of the model into treatment modalities appear very limited. A stress reduction protocol based on physiological biofeedback may therefore be a useful additive for the physiotherapist. The additional value of HCT in patients with CLBP combined with a back school (BS), however, is unclear and has not been reported upon before.
The primary objective of this study was to test on which variables HCT may be additive to patients who were admitted to BS compared to patients following BS solely. The secondary objective was to test the relationships between HCT at discharge with pain, disability and health perception. It was hypothesized that higher change in $\mathrm{HC}$ score was related to change in pain, disability and health perception. The tertiary objective was to explore if differences in patient baseline characteristics were related to the outcomes in order to gain more insight in who might benefit most from the addition of HCT.

\section{Methods}

Design

A retrospective cohort study was carried out, based on clinical data derived from 2008 to 2013 from patients with baseline and discharge scores. Two groups were compared, a BS + HCT group (BS-HCT) and the BS only group (BS). Because this was a pragmatic trial carried out in a care as usual program, patients and therapists were not blinded.

\section{Patients}

Participants were recruited from patients with CLBP who were admitted for outpatient pain rehabilitation in a university hospital rehabilitation center in the north of the Netherlands. All patients sign informed consent for the use of their data. The inclusion criteria for BS were: non-specific CLBP lasting $>3$ months, age $>18$ years. Exclusion criteria were: mental (e.g. major psychiatric disorders) or physical causes (e.g. cardiac or pulmonary disorders or use of heart medication) or being currently treated for such. Additional criteria for HCT were: patients reported difficulties in emotion regulation or in stress coping. Criteria were verified by experienced physiotherapists who were also experienced heart coherence trainers. A rehabilitation physician approved each participant's inclusion. Because data was derived form care as usual, approval from an ethical board was not needed.

\section{Procedure}

At baseline assessment prior to treatment (T0), and at discharge (T1) patients filled out a comprehensive set of questionnaires including demographics, the Pain Disability Index (PDI), the Roland Morris Disability Questionnaire (RMDQ), Numeric Rating Scale (NRS-pain) and the Rand36. HCT was evaluated by a standardized test procedure prior HCT and at discharge by a 5 min test. Time between 
T0 and the start of treatment varied because of differences in waiting list for treatment (varying between 1 and 3 months).

\section{Intervention}

\section{Back School}

Back school was provided by experienced physiotherapists all working in a pain rehabilitation team. BS were provided on an individual basis. It focused on physical aspects, such as increase in physical capacity or ergonomics, and behavioral aspects which could be cognitive behavioral or acceptance based. In all aspects, exposure therapy was a treatment modality. The duration was 12 weeks, two times per week in a cardio fitness setting (a total of $24 \mathrm{~h}$ in the BS group).

\section{HCT}

HCT was provided 6 times, once per week in an individual setting $1 \mathrm{~h}$ per meeting extra (total $6 \mathrm{~h}$ ). HCT followed a standardized stress reduction program which was based on heart rate biofeedback supplied by the HeartMath Institute (HeartMath 2004). All training was provided by HCTlicensed physiotherapists. Two components within HCT were mindfulness and acceptance and commitment therapy (ACT). These components have a focus on exposing to feelings and thoughts as they are, without avoiding emotions including pain. The first HCT sessions were focused on training of techniques with regards to breathing en changing heart coherence. Basic techniques applied were called the 'Neutral technique' and the 'Quick Coherence technique'. After the patients mastered the basic techniques, in the second part of the training, patients were exposed to stress reactions, by focusing on individualized negative feelings and emotions (including their pain). This was done by the 'Freeze-frame' technique and mindfulness exercises. Patients were thought to focus on their body signals in stressful situations. Patients trained in a therapy setting and at home.

\section{Measurements}

\section{Pain}

Current pain intensity was measured with an 11-point numeric rating scale (NRS), ranging from 0 (no pain) to 10 (worst pain ever). Reliability and validity of the pain NRS is sufficient (Jensen et al. 1986). Minimal clinically important change (MCIC) varies from 2.5 to 4.5 points in patients with CLBP (van der Roer et al. 2006).
Disability

Roland Morris Disability Questionnaire (RMDQ) The RMDQ contains 24 questions selected from the Sickness Impact Profile. Questions reflect the areas of greatest relevance to back pain (Roland and Morris 1983). The questionnaire is constructed with dichotomous items (yes/ no) and total range varies from 0 to 24 with higher scores reflecting higher disability. The validity, reliability and responsiveness has been shown adequate (Roland and Morris 1983). MCIC is 5.0 points (Yelland et al. 2011).

Pain Disability Index (PDI) The PDI is a 7-item questionnaire to investigate the magnitude of the self-reported disability in different situations such as work, leisure time, ADL and sports. The questionnaire is constructed on an 11-item numeric rating scale (NRS) and can be considered as an interval scale in which 0 means no disability and 10 maximum disability. Reliability is moderate to good (Tait et al. 1990). MCIC is 8.5-9.5 points in patients with CLBP (Soer et al. 2012).

\section{Health Status}

Self-reported health status was measured with the Rand-36. The Rand-36 is a generic health questionnaire covering nine domains of self-reported health. Each scale can range from 0 to 100 , with higher scores indicating better health status. The validity and reliability is good (Zee van der and Sanderman 1993). MCIC of the Rand-36 is unknown but is supposed to be closely related to the MCIC of the Short Form-36, which was reported as 5.0 points in postoperative patients (Busija et al. 2008).

\section{Heart Coherence Score}

Heart rate variability (HRV) was measured with an ear sensor and gave real time feedback with the use of a hardware/software system that teaches techniques to create an optimal state in coherency of heart rate (EmWave desktop). Patients can watch their HRV on computer screen. Based on the HRV, a coherence score was calculated. Coherence was calculated by the computer software as the ratio between low frequency HRV (around 0.1 Hertz) divided by the sum of the high frequency $(0.15-0.4$ Hertz) and very low frequency $(<0.04$ Hertz) (McCraty and Watkins 1996). Coherence scores were plotted by the EmWave desktop by filling three boxes on screen. A green box indicates the time when subjects are coherent, a blue box indicates the time that patients are medium coherent, and a red box indicates time of incoherency. The sum of these boxes represent $100 \%$ of the time. An outcome measure was created by summing the blue and green boxes 
(red $\times 0$, blue $\times 1$; green $\times 2$ ). This resulted in an average weighted coherence score (HC-score) ranging from 0 to 200 with larger numbers reflecting higher coherency (Kleen and Reitsma 2011). The exact algorithms for the translation of the HRV frequencies to coherent scores are performed by the software of the developers and are unknown to the researchers. HC-score was acquired during a 5 min measurement on level 2 (standard level provided and recommended for scientific measurements by the Heartmath Institute) (HeartMath 2004).

\section{Statistics}

First, baseline characteristics were tested on difference between HCT Group and BS with Mann-Whitney (ordinal data) or Cramér's V test (dichotomous data). To test differences over time and between group, within and between group analyses were performed. Data were checked on normality with the Shapiro-Wilk test. Based on normality, parametric or non-parametric tests were used. For within group analyses, these were dependent $t$ tests or Wilcoxon matched pairs test. Effect sizes (ES) were calculated with normal distributed data and were interpreted as follows: ES $\leq 0.20$ is small effect; 0.50 moderate effect and $>0.80$ is large effect (Cohen 1988). For between group analyses Levene's test for equality of variances were applied. Between group analyses were performed with independent $t$ tests or Mann-Whitney U tests. Both means ( \pm standard deviation) and medians ( \pm interquartile range) are presented. Because this was a hypothesis generating study in which no a priory hypothesis were made, a Bonferroni correction was applied by dividing the significance level by the number of tested hypotheses $(0.05 / 12=0.004)$.

To test if change over time in heart coherence score $(\Delta$ $\mathrm{HC}$ score) was related to a larger difference in disability, health status or pain, Pearson correlation coefficients (r) were used. Correlations ranging from 0.00 to 0.25 indicate little or no correlation; from 0.26 to 0.50 indicate a fair correlation; values of 0.51 to 0.75 are moderate to good; values above 0.75 are considered good to excellent (Portney and Watkins 2000). Missing data were handled as follows: for the RMDQ and PDI, when a maximum of two items were missing, scores were replaced by the case series mean. If more items were missing, the RMDQ was excluded from further analyses.

To study which baseline characteristics of patients may relate to a higher $\Delta \mathrm{HC}$ score a linear regression analysis was performed with gender, age, baseline pain intensity and baseline RMDQ score as independent variables and $\Delta$ HC score as dependent variable. $p$ values $<0.05$ were considered statistically significant. All analyses were performed with SPSS-20.
Table 1 Baseline characteristics

\begin{tabular}{llll}
\hline & HCT-group & BS-group & $p$ value \\
\hline $\mathrm{N}$ & 81 & 89 & \\
Age (years $\pm \mathrm{SD})$ & $43.7 \pm 12.9$ & $44.8 \pm 14.1$ & 0.60 \\
Gender male/female $(\mathrm{N})$ & $40 / 41$ & $59 / 30$ & $0.03^{*}$ \\
\hline
\end{tabular}

$N$ number of patients, $S D$ standard deviation

$* p<0.05$

\section{Results}

Subjects

In Total, 170 patients were included in this study $(81 \mathrm{HCT}$; $89 \mathrm{BS})$. Mean age of HCT group was $43.7 \pm(12.9)$ and $44.8( \pm 14.1)$ years in the BS group. There was a significant difference in gender distribution between groups. Age was not significantly different between groups. Characteristics of patients are presented in Table 1.

\section{Outcomes}

Pain intensity (NRS), PDI and subscales of the Rand-36, social functioning, role limitations physically and emotionally, health perception and health change were not normally distributed and were tested with a Wilcoxon matched-pairs test. Both groups significantly improved on NRS pain, RMDQ, PDI and sub-scales physical functioning, social functioning, role limitations physical, vitality, pain and health change of the Rand-36 (Table 2). There were large ES in the BS-HCT group for the sub-scale physical functioning and the RMDQ. There were moderate ES for the BS group. Both groups had large ES on sub-scale pain of the Rand-36. Compared to BS, the BS-HCT group performed significantly better on physical functioning of the Rand-36 $(p=0.02)$, but not on the other outcomes.

Significant changes $(p<0.01)$ were observed in heart coherence score between T0 and T1 (Table 3). Significant moderate correlations were present between a $\Delta \mathrm{HC}$ score and $\Delta$ RMDQ $(p=0.01)$ and $\Delta$ PDI $(p=0.05)$. A nonsignificant moderate relation was present between $\Delta$ HC score and $\Delta$ sub-scale mental health of the Rand-36. In Table 4 , results of the regression analysis are presented. There were no significant relations between a $\Delta \mathrm{HC}$ score and gender, age, baseline pain intensity and baseline RMDQ (see Table 4).

\section{Discussion}

The objectives of this study were to explore if there were differences between patients with CLBP following a BS 


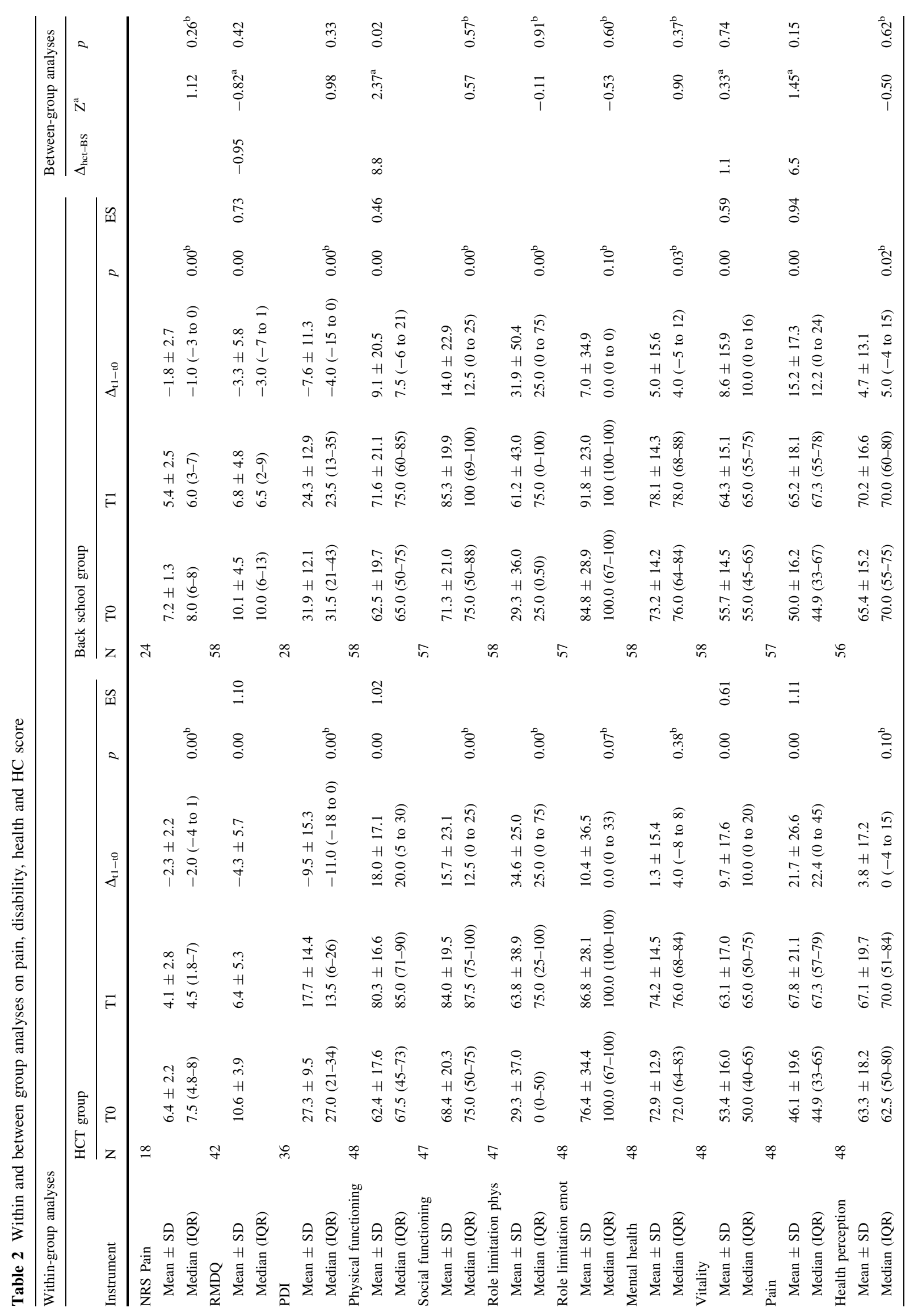




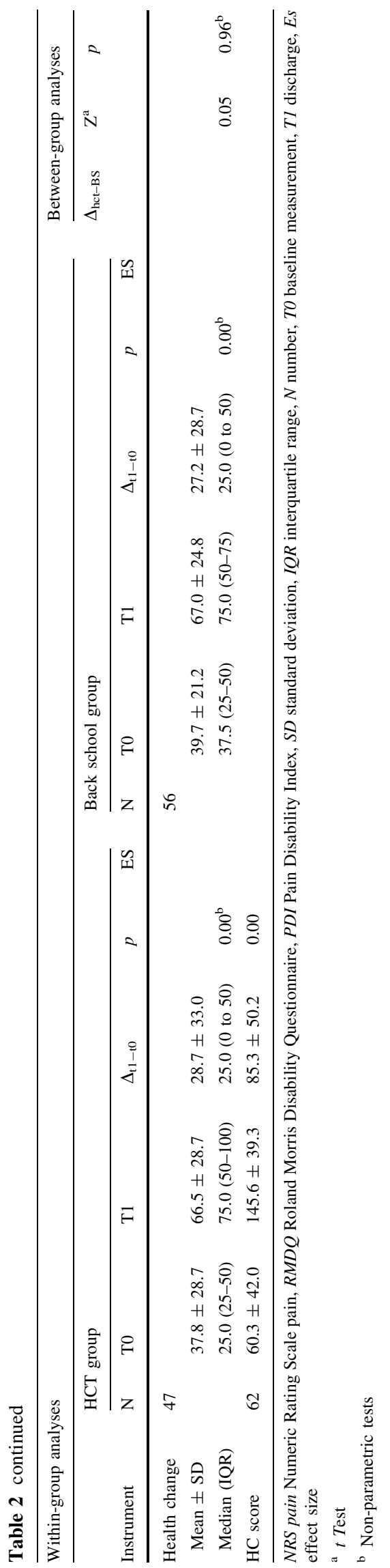

after an intervention with HCT compared to patients receiving BS only and to explore which patient characteristic are related to a higher change on $\mathrm{HC}$ score. Both groups showed significant differences on pain, disability and health status. HCT group changed significantly and relevantly more on physical functioning. Other measures were not significant, but trends can be observed in favor of HCT on outcome sub-scale pain of the Rand-36 ( $p=0.15$; $42 \%$ additional pain reduction) and NRS pain $(p=0.26$; $28 \%$ additional pain reduction). Because this study reports on first clinical results, multiple explorative hypothesis were tested, and after Bonferroni correction, no differences remained significant between the groups. Based on data from this study, more specific hypotheses can be formed. Additionally, a larger change score on HCT was related to a larger change in disability, measured with the PDI and RMDQ, but not on pain intensity. This appears in line with others (Gockel et al. 2008; Kang et al. 2012), who also reported significant correlations between a lower HRV and higher reported disability in patients with CLBP and chronic neck pain respectively but not with higher pain.

In literature, there appears conflicting evidence concerning effects of HCT on health, functioning and pain. Houtveen et al. (2012), described that heart rate variability (HRV) is likely to be related to physical and mental health, but that this, among other factors such as genetic factors, medication and sports status are important determinants for larger HRV. According to the hypothesis in the current study, physical functioning increased after HCT clinically relevant with 8.9 points more compared to the BS group, which is higher than the MCIC of 5.0 points. For mental health, a moderate but non-significant correlation was observed in the current study. Additionally, non-significant but clinically relevant changes were found on the sub-scale pain with 6.5 points, which exceeds the MCIC of 5.0 points.

Multiple reviews conclude that behavioral therapy, cognitive or acceptance based interventions are effective for patients with CLBP, but it is insufficiently studied, which elements of these interventions are effective. Physical training was found to have additionally value, and was observed non-inferior compared to physical training combined with cognitive behavioral therapy (Smeets et al. 2008). Within the context of the current study, exposure to avoidance was applied within a cardio fitness setting in which patients were exposed to avoided activities and within HCT, exposure was applied to different kinds of stress reactions. In this, a complete program was offered for physical and emotional stress reactions. Physiotherapists may benefit from more clear stress reduction programs.

The mechanisms behind heart coherence remain controversial. After training, patients significantly improved in 
Table 3 Pearson correlations between change score of heart coherence and change scores on RMDQ, PDI, NRS pain and subscales of the Rand-36

\begin{tabular}{lcc}
\hline & \multicolumn{2}{c}{$\Delta$ HC score } \\
\cline { 2 - 3 } & $\mathrm{r}$ & $\mathrm{N}$ \\
\hline NRS pain & 0.06 & 13 \\
RMDQ & $0.48^{* *}$ & 29 \\
PDI & $0.39^{*}$ & 26 \\
Rand-1 & -0.12 & 34 \\
Rand-2 & 0.05 & 34 \\
Rand-3 & 0.05 & 34 \\
Rand-4 & 0.00 & 34 \\
Rand-5 & 0.32 & 34 \\
Rand-6 & 0.09 & 34 \\
Rand-7 & -0.13 & 34 \\
Rand-8 & -0.11 & 34 \\
Rand-9 & 0.00 & 33 \\
\hline
\end{tabular}

$\triangle H C$ score change score heart coherence, NRS pain Numeric Rating Scale pain, $R M D Q$ Roland Morris Disability Questionnaire, $P D I$ Pain Disability Index

Subscales Rand-36: 1 = physical functioning, $2=$ social functioning, $3=$ role limitations physically, $4=$ role limitations emotionally, $5=$ mental health, $6=$ vitality, $7=$ pain, $8=$ health perception, $9=$ health change

$* p \leq 0.05, * * p \leq 0.01$

Table 4 Regression analyses between $\Delta \mathrm{HC}$ score and age, gender, baseline pain intensity and RMDQ score

\begin{tabular}{lrcc}
\hline Variable & \multicolumn{1}{c}{$\mathrm{B}$} & Standard error & $p$ value \\
\hline Constant & 11.9 & 44.3 & 0.79 \\
Gender $(0=$ male $)$ & 0.0 & 18.2 & 1.00 \\
Age & 0.6 & 0.8 & 0.41 \\
Pain intensity & 6.2 & 5.0 & 0.22 \\
RMDQ score & 0.6 & 2.0 & 0.76 \\
$\mathrm{R}^{2}$ & 0.10 & & \\
\hline
\end{tabular}

heart coherence score $(p<0.01)$, meaning that the technique could be adopted within six sessions and was related to change in disability. Slow breathing was previously described to be positively related to increase of HRV and HCT (Berntson et al. 1993), but the relations between slow breathing and sympatic regulation and autonomic balance remain insufficiently clear.

This appears to be the first retrospective study in which effectiveness of HCT combined with a BS was studied and compared to a care as usual program in patients with CLBP. Standardized and validated outcome measures were used and 170 patients were included in this study which contributes to generalization of results. HCT could be implemented well and was applied to good satisfaction of patients. Patients rated this 8.5 points on a $0-10$ scale (data not published).

Because this are the first explorative pragmatic results, there are methodological weaknesses in this study, which makes the evidence of this study a level $\mathrm{C}$ with high risk of bias. The first concerns the design. A retrospective study was used and patients were included based on indication by experienced physiotherapists and rehabilitation physician. This may have led to selection bias and the percentage of included women in the HCT group was higher than of men. Additionally, while the criteria for inclusion have been described, they are based on non-transparent measures of which validity is unknown. Another weakness was that only patients were included who filled out the discharge measurement, which may have led to inclusion bias. Additionally, there are no long term follow-up measures which are desirable. Another point which should be addressed in future studies is the fact that 6 extra hours of care was provided. There was no placebo group and it is known that interaction of patient and therapists is an important factor for treatment results. It is currently also unknown if the costs associated with the 6 extra hours are worth the 'benefit'. Additionally, there was an attrition bias because of substantial missing data.

Further research is necessary to gain higher quality of evidence and gain more insight in the mechanisms, mediators and moderators of HCT. The questions, what works, why does it work and for whom are still insufficiently clear. Prospective, randomized placebo controlled studies are recommended with a long term follow-up. A post hoc sample size calculation for a superiority randomized controlled trial $(\alpha=0.05$ and $\beta=0.2)$ based on the change scores of both groups on physical functioning leads to a sample size of 58 subjects per treatment arm. When correction for a $10 \%$ drop out percentage is applied, then 128 subjects need to be included in a trial. To test for whom the addition of HCT may be most beneficial, other variables are needed than those tested in this study. We advise to explore stress related variables for this.

\section{Conclusion}

A BS program based on behavioral principles and exercise therapy combined with Stress reduction based on heart coherence training may be additive on factors physical functioning and disability. Criteria for success were not identified from baseline variables. This study provides level $\mathrm{C}$ evidence that heart coherence training is effective combined with BS in patients with chronic low back pain. Prospective, randomized placebo controlled studies are recommended with a long follow-up term. 
Conflict of interest None.

Ethical standard This study was performed within a care as usual setting.

\section{References}

Agelink, M. W., Boz, C., Ullrich, H., \& Andrich, J. (2002). Relationship between major depression and heart rate variability: Clinical consequences and implications for antidepressive treatment. Psychiatry Research, 113(1-2), 139-149.

Bekkering, G. E., Hendriks, H. J. M., Koes, B. W., Oostendorp, R. A. B., Ostelo, R. W. J. G., Thomassen, J., et al. (2005). KNGF richtlijn Lage-rugpijn. No. 115.

Berntson, G. G., Cacioppo, J. T., \& Quigley, K. S. (1993). Respiratory sinus arrhythmia: Autonomic origins, physiological mechanisms, and psychophysiological implications. Psychophysiology, 30(2), 183-196.

Breivik, H., Collett, B., Ventafridda, V., Cohen, R., \& Gallacher, D. (2006). Survey of chronic pain in Europe: Prevalence, impact on daily life, and treatment. European Journal of Pain (London, England), 10(4), 287-333.

Busija, L., Osborne, R. H., Nilsdotter, A., Buchbinder, R., \& Roos, E. M. (2008). Magnitude and meaningfulness of change in SF-36 scores in four types of orthopedic surgery. Health and Quality of Life Outcomes, 6, 55.

Cohen, J. (1988). Statistical power analysis for the behavioral sciences (2nd ed.). New Jersey: Hillsdale.

Dekker, J. M., Crow, R. S., Folsom, A. R., Hannan, P. J., Liao, D., Swenne, C. A., et al. (2000). Low heart rate variability in a 2-minute rhythm strip predicts risk of coronary heart disease and mortality from several causes: The ARIC Study. Atherosclerosis Risk in Communities. Circulation, 102(11), 1239-1244.

Gockel, M., Lindholm, H., Niemisto, L., \& Hurri, H. (2008). Perceived disability but not pain is connected with autonomic nervous function among patients with chronic low back pain. Journal of Rehabilitation Medicine: Official Journal of the UEMS European Board of Physical and Rehabilitation Medicine, 40(5), 355-358.

Goedhart, A. D., Willemsen, G., Houtveen, J. H., Boomsma, D. I., \& De Geus, E. J. (2008). Comparing low frequency heart rate variability and preejection period: Two sides of a different coin. Psychophysiology, 45(6), 1086-1090.

Hallman, D. M., \& Lyskov, E. (2012). Autonomic regulation, physical activity and perceived stress in subjects with musculoskeletal pain: 24-hour ambulatory monitoring. International Journal of Psychophysiology: Official Journal of the International Organization of Psychophysiology, 86(3), 276-282.

Hallman, D. M., Olsson, E. M., von Scheele, B., Melin, L., \& Lyskov, E. (2011). Effects of heart rate variability biofeedback in subjects with stress-related chronic neck pain: A pilot study. Applied Psychophysiology and Biofeedback, 36(2), 71-80.

Hassett, A. L., Radvanski, D. C., Vaschillo, E. G., Vaschillo, B., Sigal, L. H., Karavidas, M. K., et al. (2007). A pilot study of the efficacy of heart rate variability (HRV) biofeedback in patients with fibromyalgia. Applied Psychophysiology and Biofeedback, $32(1), 1-10$

Hay, E. M., Mullis, R., Lewis, M., Vohora, K., Main, C. J., Watson, P., et al. (2005). Comparison of physical treatments versus a brief pain-management programme for back pain in primary care: A randomised clinical trial in physiotherapy practice. Lancet, 365(9476), 2024-2030.

HeartMath. (2004). emWave stress relief system.

Heimer, L., \& Van Hoesen, G. W. (2006). The limbic lobe and its output channels: Implications for emotional functions and adaptive behavior. Neuroscience and Biobehavioral Reviews, 30(2), 126-147.

Houtveen, J. H., Hornsveld, H. K., van Trier, J., \& van Doornen, L. J. (2012). Questioning the mechanism behind slow breathing and heart coherence training (Vraagtekens bij het werkingsmechanisme van slow-breathing en hartcoherentietraining). Tijdschrift Voor Psychiatrie, 54(10), 879-888.

Jensen, M. P., Karoly, P., \& Braver, S. (1986). The measurement of clinical pain intensity: A comparison of six methods. Pain, 27(1), 117-126.

Kang, J. H., Chen, H. S., Chen, S. C., \& Jaw, F. S. (2012). Disability in patients with chronic neck pain: Heart rate variability analysis and cluster analysis. The Clinical Journal of Pain, 28(9), 797-803.

Keefe, F. J., Rumble, M. E., Scipio, C. D., Giordano, L. A., \& Perri, L. M. (2004). Psychological aspects of persistent pain: Current state of the science. The Journal of Pain: Official Journal of the American Pain Society, 5(4), 195-211.

Kleen, M., \& Reitsma, B. (2011). Appliance of heart rate variability biofeedback in acceptance and commitment therapy: A pilot study (null). Journal of Neurotherapy, 15(2), 170-181.

Lane, R. D., McRae, K., Reiman, E. M., Chen, K., Ahern, G. L., \& Thayer, J. F. (2009). Neural correlates of heart rate variability during emotion. NeuroImage, 44(1), 213-222.

Lehrer, P. M., Vaschillo, E., Vaschillo, B., Lu, S. E., Eckberg, D. L., Edelberg, R., et al. (2003). Heart rate variability biofeedback increases baroreflex gain and peak expiratory flow. Psychosomatic Medicine, 65(5), 796-805.

Lemaire, J. B., Wallace, J. E., Lewin, A. M., de Grood, J., \& Schaefer, J. P. (2011). The effect of a biofeedback-based stress management tool on physician stress: A randomized controlled clinical trial. Open Medicine: A Peer-Reviewed, Independent, OpenAccess Journal, 5(4), e154-e163.

McCraty, R. (2001). Science of the heart: Exploring the role of the heart in human performance [null]. Boulder Creek, CA: HearthMath Research Center.

McCraty, R., Atkinson, M., Tomasino, D., \& Trevor Bradley, R. (2009). The coherent heart: Heart-brain interactions, psychophysiological coherence, and the emergence of system-wide order. HeartMath Research Center, 5(2), 10-115.

McCraty, R., \& Tomasino, D. (2006). Emotional stress, positive emotions, and psychophysiological coherence. In B. B. Arnetz \& R. Ekman (Eds.), Stress in health and disease (pp. 342-365). Germany: Weinheim.

McCraty, R., \& Watkins, A. D. (1996). Autonomic assessment report: A comprehensive heart rate variability analysis. Boulder Creek, CA: Institute of Heart Math.

Portney, L. G., \& Watkins, M. P. (2000). Foundations of clinical research: Applications to practice (2nd ed.). Upper Saddle River: Prentice Hall.

Roland, M., \& Morris, R. (1983). A study of the natural history of back pain: Part I: Development of a reliable and sensitive measure of disability in low-back pain. Spine, 8(2), 141-144.

Smeets, R. J., Vlaeyen, J. W., Hidding, A., Kester, A. D., van der Heijden, G. J., \& Knottnerus, J. A. (2008). Chronic low back pain: Physical training, graded activity with problem solving training, or both? The 1-year post-treatment results of a randomized controlled trial. Pain, 134(3), 263-276.

Soer, R., Reneman, M. F., Vroomen, P. C., Stegeman, P., \& Coppes, M. H. (2012). Responsiveness and minimal clinically important change of the Pain Disability Index in patients with chronic back pain. Spine, 37(8), 711-715.

Stein, P. K., \& Kleiger, R. E. (1999). Insights from the study of heart rate variability. Annual Review of Medicine, 50, 249-261.

Taelman, J., Vandeput, S., Gligorijevic, I., Spaepen, A., \& Van Huffel, S. (2011). Time-frequency heart rate variability 
characteristics of young adults during physical, mental and combined stress in laboratory environment. In Conference proceedings: Annual international conference of the IEEE engineering in medicine and biology society (pp. 1973-1976). IEEE.

Tait, R. C., Chibnall, J. T., \& Krause, S. (1990). The Pain Disability Index: Psychometric properties. Pain, 40(2), 171-182.

Tulder, M. W. v., Custers, J. W. H., Bie, R. A. d., Hammelburg, R., Hulshof, C. T. J., Kolnaar, B. G. M., et al. (2010). Ketenzorgrichtlijn Aspecifieke Lage Rugklachten.

Tunks, E. R., Crook, J., \& Weir, R. (2008). Epidemiology of chronic pain with psychological comorbidity: Prevalence, risk, course, and prognosis. Canadian Journal of Psychiatry. Revue Canadienne De Psychiatrie, 53(4), 224-234.

Turk, D. C., \& Okifuji, A. (2002). Psychological factors in chronic pain: Evolution and revolution. Journal of Consulting and Clinical Psychology, 70(3), 678-690.

van der Roer, N., Ostelo, R. W., Bekkering, G. E., van Tulder, M. W., $\&$ de Vet, H. C. (2006). Minimal clinically important change for pain intensity, functional status, and general health status in patients with nonspecific low back pain. Spine, 31(5), 578-582. van der Zee, K. I., \& Sanderman, R. (1993). Het meten van de algehele gezondheidstoestand met de RAND-36. Article in Dutch. Groningen, The Netherlands: Noordelijk Centrum voor Gezondheidsvraagstukken. Rijksuniversiteit Groningen.

van Middendorp, H., Lumley, M. A., Houtveen, J. H., Jacobs, J. W., Bijlsma, J. W., \& Geenen, R. (2013). The impact of emotionrelated autonomic nervous system responsiveness on pain sensitivity in female patients with fibromyalgia. Psychosomatic Medicine.

Van Oosterwijck, J., Nijs, J., Meeus, M., \& Paul, L. (2013). Evidence for central sensitization in chronic whiplash: A systematic literature review. European Journal of Pain (London, England), 17(3), 299-312.

Yelland, M., Hooper, A., \& Faris, P. (2011). Minimum clinically important changes in disability in a prospective case series with chronic thoracic and lumbar spinal pain. International Musculoskeletal Medicine, 33(2), 49-53. 